'Sección Medicina Nuclear, Departamento de Medicina, Hospital Clínico Universidad de Chile.

${ }^{2}$ Centro PET de Imágenes Moleculares, Hospita Militar de Santiago, HOSMIL Chile.

${ }^{3}$ Servicio de Medicina Nuclear, Hospital Dirección de Previsión de Carabineros de Chile, DIPRECA.

${ }^{4}$ Departamento Anatomía Patológica, Hospital Clínico Universidad de Chile.

Recibido el 5 de enero de 2012, aceptado el 23 de mayo de 2012.

Correspondencia a: Dra. Teresa Massardo Vega Sección Medicina Nuclear, Depto. Medicina Hospital Clínico Universidad de Chile.

Santos Dumont 999-1E Independencia, Santiago Chile. Fono: 562-7770569 Fax: 562-7777618 E-mail: tmassardo@ redclinicauchile.c. centropet@hosmil.c

\section{Utilidad del estudio PET con FDG en la evaluación de sarcomas de diverso origen y de tumores no sarcoma-no epiteliales}

\author{
TERESA MASSARDO ${ }^{1,2}$, MARÍA JOSEFINA JOFRÉ ${ }^{2}$, \\ MARÍA PAULINA SIERRALTA ${ }^{2}$, JOSÉ CANESSA ${ }^{2,3}$, \\ GABRIEL CASTRO $^{1}$, ISABEL BERROCAL ${ }^{1}$, IVÁN GALLEGOS ${ }^{4}$
}

Background: The usefulness of positron emission tomography (PET) with fluorine-deoxyglucose (FDG) in sarcomas and non-sarcoma non-epithelial (NSNE) tumors is not clearly defined. Aim: To report a Chilean experience with NSNE tumors evaluated using PET with FDG. Material and Methods: Retrospective review of the database of a PET laboratory. Demographic data, indications and metabolic findings were compared with conventional imaging in 88 adults and children with diverse bone and soft tissue sarcomas as well as 24 gastrointestinal stromal tumors (GIST), 6 pleural malignant mesotheliomas in adults, and 9 medulloblastomas in children. Results: FDG showed good concordance with conventional imaging in NSNE tumors. It was helpful for staging, restaging, follow-up after treatment and for the detection of new not previously suspected lesions. Conclusions: PET with FDG could have a prognostic role and help in patient management, mainly in musculoskeletal and high grade or less differentiated sarcomas. In GIST, it was a good tool for immunotherapy control.

(Rev Med Chile 2012; 140: 1116-1125).

Key words: Neoplasms; Positron-emission Tomography; Sarcoma.

L a utilidad de la tomografía de positrones (PET) con flúordeoxiglucosa (FDG) está reconocida para carcinomas pulmonares, colorrectales, mamarios, esofágicos, de cabeza y cuello, tiroideos desdiferenciados y para linfomas y melanomas ${ }^{1-3}$. Los carcinomas de alto grado o menos diferenciados tienen alta utilización de glucosa y FDG, por su expresión de transportadores GLUT superficiales ${ }^{4}$. Los tumores de crecimiento lento tienen menor avidez (tiroideos diferenciados, neuroendocrinos, algunos de sistema nervioso, prostáticos o ciertos linfomas no Hodgkin); por otra parte, los sarcomas pueden presentar capta- ción variable con utilidad no bien establecida pues las publicaciones cuentan con muestras pequeñas y/o heterogéneas. Existe alto rendimiento para sarcomas óseos, pero menos información en otros tipos y localizaciones. Entre los tumores no epiteliales que no corresponden a sarcomas ni carcinomas (NSNE) se encuentran los gastrointestinales estromales (GIST), mesoteliomas malignos y meduloblastomas, altamente agresivos, los últimos clasificados como tumores embrionarios grado IV por la Organización Mundial de la Salud (OMS) ${ }^{5}$. El rendimiento del estudio con FDG en GIST es muy elevado para diagnóstico y pronóstico. El 
PET con FDG en sarcomas y tumores no sarcoma-no epiteliales - T. Massardo et al

resto de tumores NSNE está en evaluación, en parte, por su baja prevalencia. Específicamente, los sarcomas son de difícil clasificación por su diversidad, algunos de alto grado o indiferenciados no se asocian claramente con origen celular específico. Actualmente, la clasificación OMS es ampliamente aceptada ${ }^{6}$; una adaptación simple de los tumores óseos malignos incluye: cartílago, osteogénico, fibrogénicos, fibrohistiocítico, Ewing y neuroectodérmicos primitivos (PNET) que se consideran de la familia Ewing, hematopoyéticos, células gigantes, notocordales, vasculares, miogénicos, lipogénicos y epiteliales; de tejidos blandos incluye: adipocíticos; fibroblásticos/miofibroblastos (fibrohistocíticos), músculo liso y esquelético, vascular, condral, óseos e inciertos ${ }^{7}$.

Las indicaciones para estudios con FDG de las guías $\mathrm{NCCN}^{8}$ corresponden a:

I. Diagnóstico / Estadiaje: a) Estadiaje auxiliar de sarcoma Ewing; b) Detección de metástasis extrapulmonares de otros sarcomas (sin compromiso pulmonar).

II. Reestadiaje / recidiva: (no claro en sarcomas). III.Pronóstico: sólo en GIST.

IV.Planificación del tratamiento y monitorización de respuesta: en GIST.

Considerando que aún existe cierto grado de controversia y poca información sobre el rendimiento del estudio con FDG en evaluación de sarcomas y tumores NSNE, el objetivo de este trabajo fue investigar su aporte en nuestra experiencia.

\section{Método}

\section{Población}

Revisamos retrospectivamente nuestra base de datos desde 2003 con más de 4.000 estudios y seleccionamos 216 estudios con sarcomas (4\%) y NSNE $(1,2 \%)$ según clasificación histológica $\mathrm{OMS}^{6}$.

A. 163 estudios PET-FDG en 87 pacientes con sarcomas. Edad variaba entre 2 y 82 años; $52 \%$ hombres; 26 pacientes con estudios seriados con FDG (mediana: 3).

B. 53 estudios en 39 pacientes con tumores NSNE, $58 \%$ hombres, entre 3 y 81 años. Correspondían a 24 GIST, 6 mesoteliomas malignos pleurales y 9 meduloblastomas (11 con 2 ó 3 estudios).

\section{Técnica}

Imágenes de cuerpo entero o cabeza y cuello en meduloblastomas con equipo PET Siemens, técnica similar a previamente descrita ${ }^{3}$. Actividad de FDG según peso en niños; $13 \pm 2$ mCi adultos (glicemia: $91 \pm 10 \mathrm{mg} / \mathrm{dl}$ ). Análisis cuantitativo estandarizado de actividad de glucosa máximo $\left(\mathrm{SUV}_{\max }\right)$. Se revisaron antecedentes e indicaciones, incluyendo estadiaje, reestadiaje y control de terapia en relación a histología. Se consignó extensión local, a distancia así como antecedente de recidiva.

\section{Estadística}

Se correlacionaron los hallazgos del estudio con FDG interpretados como malignos con antecedentes de anatomía patológica (disponibles en 41 pacientes con sarcomas) y de imágenes anatómicas concomitantes: radiografías, resonancias magnéticas (RM), tomografías computadas (TC) o cintigrafías óseas; en 22\% no estaban disponibles. Se consignaron nuevas lesiones consideradas malignas, según su aspecto imagenológico en el estudio FDG. Hubo escasa disponibilidad de confirmación histológica en hallazgos de nuevas lesiones secundarias. Se efectuó concordancia con kappa de Cohen para muestras pequeñas excluyendo casos con seguimiento metabólico aislado.

\section{Resultados}

\section{A. Sarcomas}

Los más frecuentes fueron músculo-esqueléticos y luego partes blandas. En adultos, la distribución según histología y datos demográficos están en Tabla 1. La Figura 1 muestra distribución según edad. Del total, $47 \%$ correspondió a extremidades, $38 \%$ cuerpo y $15 \%$ cabeza o cuello. Los niños con sarcomas fueron $22 \%$ (26\% óseos, incluyendo osteosarcomas y de familia Ewing) y $74 \%$ partes blandas (> 50\% rabdomiosarcomas); en adultos $31 \%$ eran óseos, $7 \%$ condrosinoviales y $62 \%$ partes blandas. Hubo 4 PNET en diversas partes blandas. Período entre diagnóstico y estudio con FDG: $23 \pm 31$ meses. Dos pacientes tenían otro cáncer (melanoma y linfoma no Hodgkin); dos sarcomas germinales tenían seminoma y no seminoma previos y otro metástasis hepáticas de sarcoma indiferenciado desconocido. Hubo casos no especificados en ítem NOS de sarcomas 
PET con FDG en sarcomas y tumores no sarcoma-no epiteliales - T. Massardo et al

Tabla 1. Distribución según histología, datos demográficos y principales hallazgos en sarcomas

\begin{tabular}{|c|c|c|c|c|c|c|c|c|}
\hline & n (FDG) & Hombres & $\begin{array}{l}\text { Extensión/ } \\
\text { recidiva }\end{array}$ & E/R/TC & $\begin{array}{l}\text { FDG } \\
(+)\end{array}$ & $\begin{array}{l}\text { [NL en } \\
\text { FDG }(+)]\end{array}$ & \multicolumn{2}{|c|}{$\begin{array}{l}\text { Concordancia } \\
\text { Kappa (k) }\end{array}$} \\
\hline Osteosarcoma & $13(27)$ & $62 \%$ & $54 \%$ & $1 / 17 / 9$ & $69 \%$ & [10/19] & $95 \%$ & $k=0,877$ \\
\hline Ewing o PNET & $15 \quad(29)$ & $40 \%$ & $60 \%$ & $5 / 15 / 9$ & $50 \%$ & {$[7 / 14]$} & $87 \%$ & $k=0,904$ \\
\hline Fibro/Histiocitoma & 14 (18) & $50 \%$ & $50 \%$ & $2 / 12 / 4$ & $56 \%$ & {$[6 / 10]$} & \multirow{4}{*}{$92 \%$} & \multirow{4}{*}{$k=0,853$} \\
\hline Rabdomiosarcoma & 9 (21) & $78 \%$ & $67 \%$ & $1 / 8 / 12$ & $52 \%$ & {$[6 / 11]$} & & \\
\hline Liposarcoma & $6 \quad(9)$ & $83 \%$ & $50 \%$ & $2 / 5 / 2$ & $56 \%$ & {$[3 / 5]$} & & \\
\hline Condro-/sinovial & $6 \quad(7)$ & $17 \%$ & $67 \%$ & $1 / 5 / 1$ & $57 \%$ & {$[2 / 4]$} & & \\
\hline Angiosarcoma & $5 \quad(15)$ & $60 \%$ & $80 \%$ & $2 / 4 / 9$ & $80 \%$ & {$[8 / 12]$} & \multirow{4}{*}{$93 \%$} & \multirow{4}{*}{$k=0,844$} \\
\hline Sarcoma uterino & $5 \quad(6)$ & $0 \%$ & $100 \%$ & $2 / 4 / 0$ & $83 \%$ & {$[4 / 5]$} & & \\
\hline Sarcoma alto grado & $5 \quad(19)$ & $80 \%$ & $60 \%$ & $2 / 10 / 7$ & $63 \%$ & {$[5 / 12]$} & & \\
\hline NOS/otros & 9 (12) & $55 \%$ & $55 \%$ & $2 / 5 / 5$ & $58 \%$ & [5/8] & & \\
\hline Sarcoma total & $87(163)$ & $52 \%$ & $66 \%$ & $20 / 85 / 58$ & $61 \%$ & {$[56 / 100]$} & $92 \%$ & $k=0,837$ \\
\hline
\end{tabular}

E: Estadiaje. NOS: No especificado. R: Reestadiaje/Recaída. NL: Casos nuevas lesiones malignas. TC: Control de terapia.

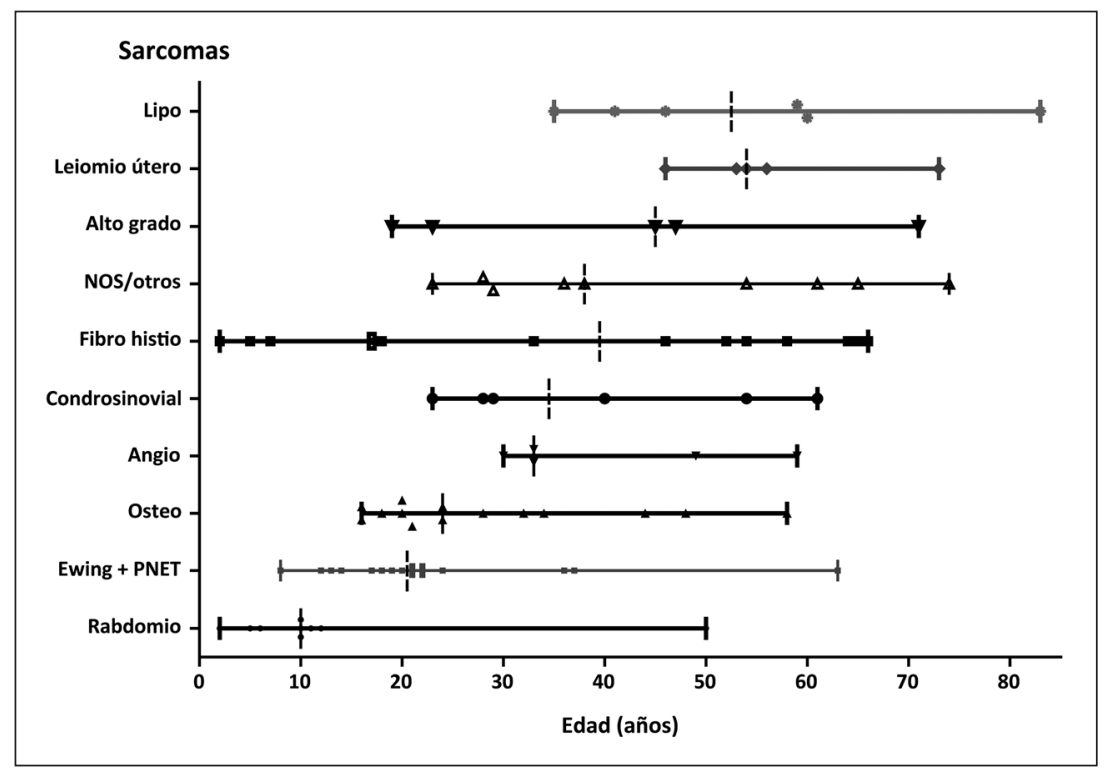

Figura 1. Distribución por edad según tipo histológico de los sarcomas (se presenta cada caso, rango y mediana). retroperitoneales, pélvico y de muslo y en ítem OTROS, se incluyó tumor embrionario, epitelioide y PEComa (mesenquimático perivascular epitelioide). Respecto a terapias, $68 \%$ tenía cirugía del primario, $47 \%$ radioterapia y $66 \%$ quimioterapia. La indicación en sarcomas fue: 12\% estadiaje, 52\% reestadiaje y $36 \%$ control de terapia. El tiempo entre diagnóstico y FDG de 0,2-2 meses en estadiaje y los ya tratados o reestadiaje entre 3 y hasta 300 meses (condrosarcoma mixoide operado, recidivado con estudio FDG positivo); los por control de terapia entre 1 y 4 meses. Veintisiete pacientes con sarcoma locomotor tenían cintigrafía ósea previa, 12 con lesiones osteoblásticas. El 60,7\% de los FDG fue positivo para lesiones malignas; se encontraron nuevas lesiones no conocidas en 


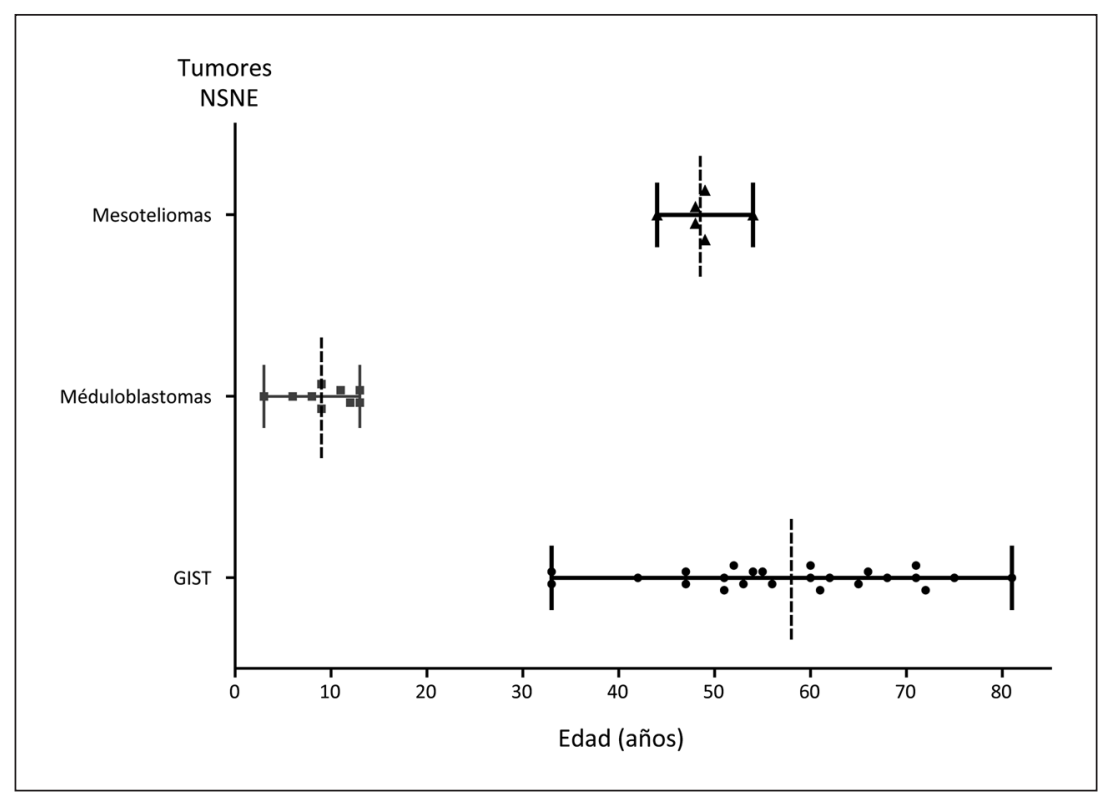

Figura 2. Distribución por edad según tipo histológico de los tumores NSNE (se presenta cada caso, rango y mediana).

Tabla 2. Distribución según histología, datos demográficos y principales hallazgos en tumores no sarcomas-no carcinomas (NSNE)

\begin{tabular}{|lrccccccc|}
\hline & n (FDG) & Hombres & $\begin{array}{c}\text { Extensión/ } \\
\text { recidiva }\end{array}$ & E/R/TC & FDG (+) & $\begin{array}{c}\text { [NL en } \\
\text { FDG(+)] }\end{array}$ & $\begin{array}{c}\text { Concordancia } \\
\text { Kappa (k) }\end{array}$ \\
inicial & & & & & & & \\
GIST & $24(34)$ & $50 \%$ & $62 \%$ & $4 / 12 / 18$ & $47 \%$ & {$[14 / 16]$} & $78 \%$ & $k=0,569$ \\
Mesotelioma & $6(6)$ & $50 \%$ & $17 \%$ & $3 / 0 / 3$ & $100 \%$ & {$[4 / 6]$} & $100 \%$ & - \\
Meduloblatoma & $9(13)$ & $78 \%$ & $78 \%$ & $0 / 6 / 7$ & $46 \%$ & {$[3 / 6]$} & $82 \%$ & $k=0,633$ \\
\hline
\end{tabular}

E: Estadiaje. R: Reestadiaje/Recidiva. TC: Control de Terapia. NL: Casos con nuevas lesiones malignas.

$67 \%$ de ellos (34\% de todos los estudios). Hubo alta concordancia con datos disponibles incluyendo imágenes anatómicas, cintigrafías óseas y biopsias, para actividad tumoral (92,4\%; kappa: $0,837)$. Figuras 3 y 4 .

\section{B. Tumores NSNE}

Incluyó a 24 pacientes con GIST entre 33 y 81 años de edad, a 6 pacientes con mesotelioma pleural maligno, entre 44 y 54 años y a 9 niños con meduloblastoma, entre 3 y 13 años. Los datos demográficos según histología, edad y resultados del estudio con FDG son mostrados en la Tabla 2 y Figura 2.

GIST: La mayoría referidos para control de terapia $(53 \%)$ que incluía inmunoterapia salvo uno por quimioterapia convencional en GIST esofágico diseminado. Su localización inicial por frecuencia fue: 9 en intestino delgado ( 3 yeyuno, 1 íleon, 1 duodeno y 1 duodenoyeyunal así como 3 no especificados), 5 gástricos, 1 esofágico, 1 vesical y los restantes fueron indeterminados a nivel abdominal o retroperitoneal. El 79\% tenían cirugía previa; 17 estudios FDG fueron realizados para valorar respuesta a terapia con Glivec $^{\circledR}$ o Sutent ${ }^{\circledR}$ (rango total de terapia: 3-43 meses). El SUV máx promedio pre terapia fue 8,5 $\pm 3,4$ y varió entre 2,5 y 13,$1 ; 2 / 4$ casos evaluados por estadiaje, sometidos recientemente a cirugía, estaban diseminados según el estudio con FDG, con focos no conocidos esqueléticos y pélvicos. Un hombre de 62 años con fibrosarcoma de pared 

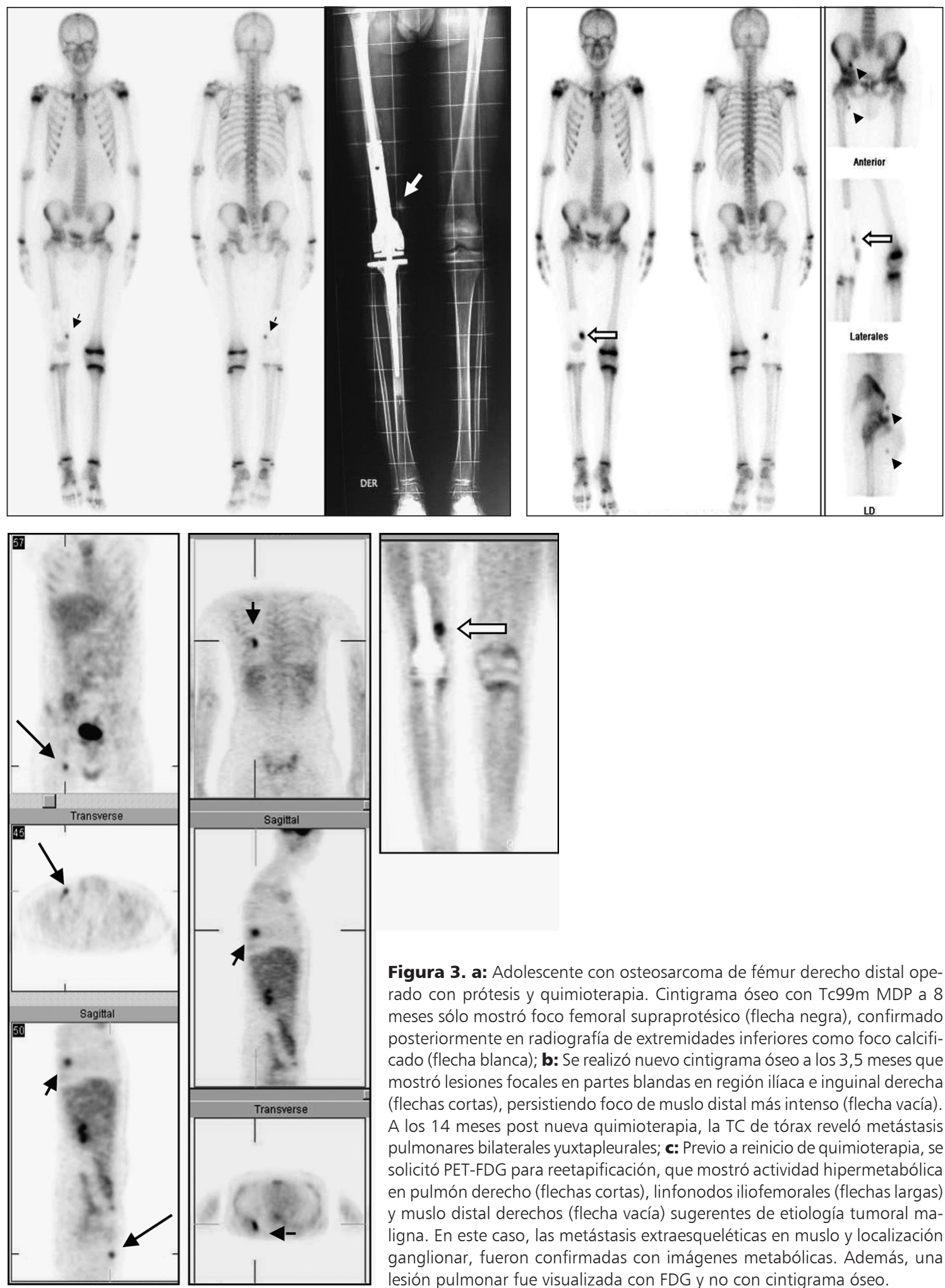

Figura 3. a: Adolescente con osteosarcoma de fémur derecho distal operado con prótesis y quimioterapia. Cintigrama óseo con Tc99m MDP a 8 meses sólo mostró foco femoral supraprotésico (flecha negra), confirmado posteriormente en radiografía de extremidades inferiores como foco calcificado (flecha blanca); b: Se realizó nuevo cintigrama óseo a los 3,5 meses que mostró lesiones focales en partes blandas en región ilíaca e inguinal derecha (flechas cortas), persistiendo foco de muslo distal más intenso (flecha vacía). A los 14 meses post nueva quimioterapia, la TC de tórax reveló metástasis pulmonares bilaterales yuxtapleurales; c: Previo a reinicio de quimioterapia, se solicitó PET-FDG para reetapificación, que mostró actividad hipermetabólica en pulmón derecho (flechas cortas), linfonodos iliofemorales (flechas largas) y muslo distal derechos (flecha vacía) sugerentes de etiología tumoral maligna. En este caso, las metástasis extraesqueléticas en muslo y localización ganglionar, fueron confirmadas con imágenes metabólicas. Además, una lesión pulmonar fue visualizada con FDG y no con cintigrama óseo. 


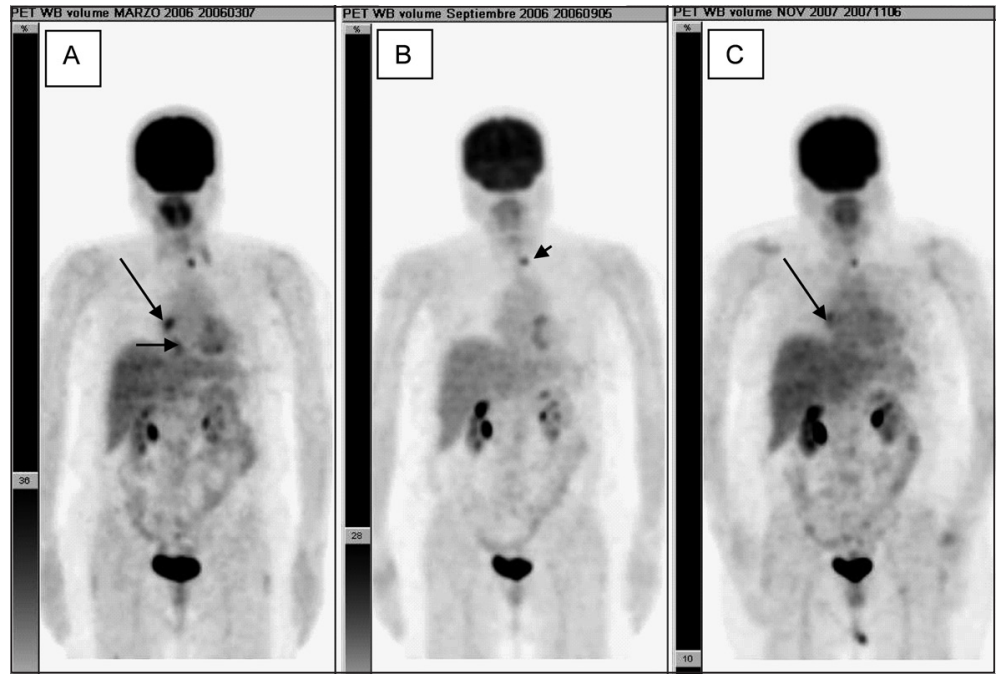

Figura 4. Mujer de 52 años con antecedente de angiosarcoma de aurícula derecha operada y tratada con QT y RT, recidivado en múltiples ocasiones. Se efectuaron estudios PET-FDG (A): que muestra actividad tumoral en dos focos pericardíacos derechos, con respuesta completa en estudio 6 meses posterior (B) y nueva recidiva 14 meses posterior a este en relación a la aurícula derecha demostrado también con FDG (C). Incidentalmente, se visualiza un nódulo tiroídeo hipermetabólico, que no varía en los tres estudios (flecha corta). abdominal resecado recientemente y con cáncer rectal operado e irradiado hace 12 años, presentaba GIST maligno vesical con infiltración de tejido adyacente e índice de proliferación elevado (10\%); tenía múltiples focos abdominales y pélvicos así como hepáticos. Entre los estudios inicialmente positivos con seguimiento con FDG para respuesta a inmunoterapia a 3 meses, un caso disminuyó $\mathrm{SUV}_{\text {max }}$ de 11,3 a negativo. Sólo 3 pacientes con inmunoterapia fueron negativos para tumor activo. Dos pacientes con regresión en TC abdominal de $20 \%$ y $50 \%$, respectivamente, tenían persistencia de actividad glucídica en masa residual y $S_{\text {máx }}$ de 4,9 y 11 , respectivamente. La concordancia con otros antecedentes fue buena aunque con kappa moderado. Figura 5.

Mesoteliomas Malignos: Todos tenían enfermedad localmente avanzada, de primarios pleurales. No hubo datos completos del tipo histológico (sarcomatoide, epitelioide o mixto). Fueron evaluados para estadiaje y tratados con cirugía o quimioterapia, uno con pleurodesis adicional con talco por derrame importante. Todos presentaban extenso e intenso hipermetabolismo pleural muy irregular; $\mathrm{SUV}_{\text {máx }}$ promedio 6,7, rango entre 3 y 8 (excluyendo la pleurodesis). Uno tenía compromiso pleural bilateral, dos extensiones peritoneales y otro compromiso multifocal esquelético. La concordancia con otros antecedentes, disponibles en todos los casos, en especial TC de tórax y abdomen, fue muy buena (muestra pequeña para kappa). Todos

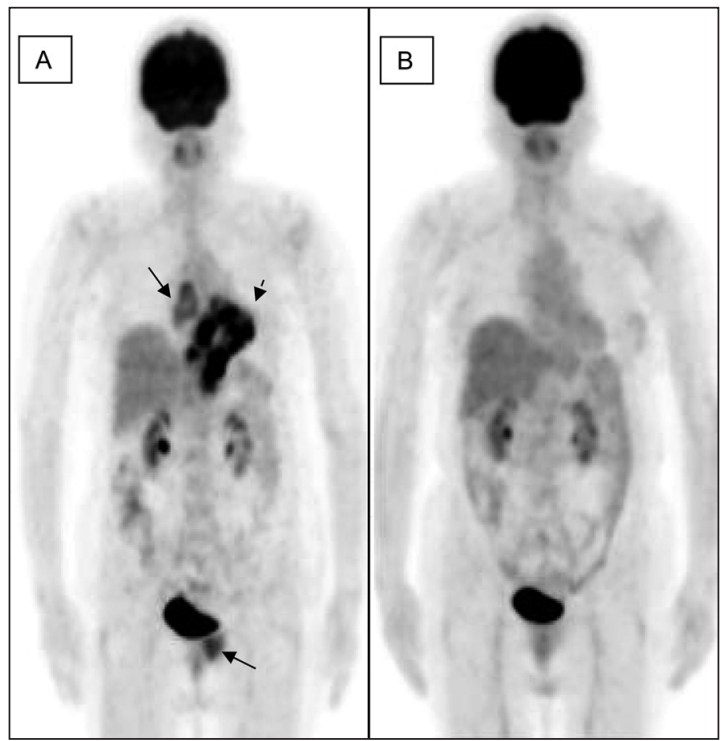

Figura 5. Mujer de 65 años, con GIST etapa IV de origen indeterminado en estadiaje. TC tórax y abdomen: tumor mediastino posterior $9 \times 8 \mathrm{~cm}$ en relación a esófago, contacta cavidades cardíacas; lesión osteolítica $9^{\circ}$ arco costal posterior izquierdo asociado a masa partes blandas de 2,5 cm; adenopatías periportales y pericavas de hasta $2 \mathrm{~cm}$. TC cerebro: $\sin$ hallazgos patológicos. Cintigrama óseo: focos osteoblásticos costal e iliopubiano izquierdos sugerentes de metástasis. Biopsia costal: tumor de células fusadas concordante con GIST. Se realiza PET-FDG basal (A) (imagen 3D en AP): gran masa hipermetabólica toracoabdominal posterior (flecha corta) y compromiso esquelético dorsal y pubiano (flechas largas). Luego de 3 meses con Glivec $\AA$, FDG control (B) muestra regresión total de las lesiones hipermetabólicas. 
PET con FDG en sarcomas y tumores no sarcoma-no epiteliales - T. Massardo et al

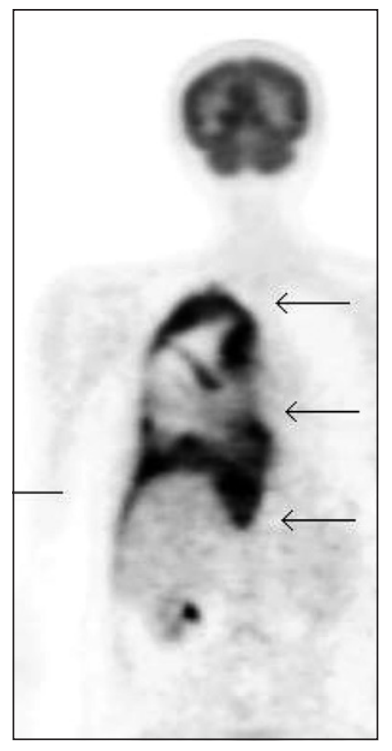

Figura 6. Paciente de 49 años de sexo masculino con extenso mesotelioma maligno pleural derecho, con antecedente de Linfoma de Hodgkin hace más de 20 años (esplenectomizado). TC de tórax: muestra engrosamiento pleural difuso a derecha con derrame encapsulado. TC abdomen y pelvis: sin alteraciones. PET-FDG para etapificación: intensa captación irregular que compromete gran parte de la superficie pleural derecha especialmente medial basal, que se extiende hacia la superficie diafragmática y peritoneal perihepática. fallecieron por su enfermedad entre $4 \mathrm{y}$ 16 meses post-diagnóstico. El estudio con FDG se efectuó entre 1 y 7 meses predeceso. Figura 6.

Meduloblastomas: Todos los niños habían sido sometidos a cirugía, radioterapia y o quimioterapia. No existía sospecha de compromiso medular ni antecedentes respecto a extensión local extracerebral salvo un caso. Los con tumor recurrente tenían captación local leve a moderada de FDG en fosa posterior; en un caso, en ambos hemisferios cerebelosos. El SUV máx $_{\text {en }}$ los cinco casos positivos varió entre 4,3 y 15,9 . La concordancia del FDG fue muy buena con ajuste kappa importante respecto a imágenes anatómicas (todos con RM cerebral previa, salvo uno sólo con TC). Cuatro pacientes tenían seguimiento con FDG; se cuentan con datos de sobrevida de siete pacientes, se conocen tres fallecimientos con recidiva tumoral tratada, uno con FDG negativo 2 meses antes, (sin causa de muerte especificada, posible falso negativo del FDG) y los otros con FDG positivo, 24 y 12 meses previo a su muerte. Figura 7.

\section{Discusión}

Los tumores malignos están au-

Figura 7. Paciente de 11 años con meduloblastoma sometido a exéresis total y tratado con radio y quimioterapia posterior. Tuvo recidiva cerebelosa izquierda operada y nueva quimioterapia. PET-FDG (A) (cortes dispuestos verticalmente: axial, sagital y coronal) muestra actividad residual cerebelosa (flecha larga). Recibe nueva RT y se realiza control a los $12 \mathrm{~m}$ con estudio FDG (B) que demuestra actividad focal aumentada en ambos hemisferios cerebelosos (flechas cortas) compatible con progresión. Fallece a los 12 días con diagnóstico de meduloblastoma recidivado en 2 oportunidades; T4 etapa M2. mentando y se ha planteado que los sarcomas probablemente tienen baja notificación en nuestro país. Sin embargo, la prevalencia y distribución por edad es similar a la reportada en Estados Unidos de Norteamérica.

Los sarcomas son ligeramente más frecuentes en mujeres Esta casuística corresponde a una muestra bastante representativa de la situación local como podría deducirse de un informe regional con distribución similar a otros trabajos, aunque los tumores de partes blandas fueron más frecuentes con proporción similar entre sexos ${ }^{9,10}$. La captación de FDG y SUV está relacionados con el grado histológico del 
tumor, celularidad, actividad mitótica, marcadores como el índice MIB-1 que detecta antígeno Ki 67, y sobreexpresión de p53; que tienen valor pronóstico $^{11}$. En sarcomas óseos, el FDG puede evaluar esqueleto en forma similar al cintigrama óseo y pesquisar lesiones a distancia y en partes blandas ${ }^{12,13}$. En quimioterapia neoadyuvante, un SUV $<2,5$ se asocia a mejoría de sobrevida en osteosarcomas, correlacionándose parcialmente con respuesta histológica ${ }^{14}$; en tumores de tejidos blandos, tiene muy buena capacidad discriminativa para lesiones primarias y recurrentes con posible papel en la clasificación tumoral ${ }^{15,16}$. Sin embargo, tumores de bajo grado y lesiones benignas pueden presentar captación relativamente alta de FDG (ej. traumáticas, osteomielitis, enfermedad de Paget, condroblastoma, tumor de células gigantes). En lesiones pulmonares de sarcomas, la TC de tórax es superior a FDG. Por otra parte, no hay problemas para evaluar pacientes con prótesis.

En general, para osteosarcomas la sensibilidad es $91 \%$ y la especificidad $85 \%$. Específicamente, en familia Ewing y PNET la adición de imágenes híbridas con TC a las tradicionales con PET efectuado con FDG es importante (sensibilidad, especificidad y precisión de PET: 71\%, 95\% y $88 \%$, vs híbrido: $87 \%$, 97\% y $94 \%$, respectivamente; ambas metodologías tienen relevancia e impacto en la estrategia de tratamiento ${ }^{15,17}$. En sarcomas óseos, uno de los factores pronósticos más importantes es la respuesta a la terapia que puede ser medida como porcentaje de necrosis y se puede observar como hipometabolismo central $^{13}$. Sin embargo, la viabilidad tumoral podría ser confundida con tejido inflamatorio reactivo; por otra parte, el momento de realizar el control con FDG post-terapia está en discusión: algunos prefieren evaluar tres meses post radioterapia y 1 mes postcirugía o quimioterapia. El FDG ayuda a: confirmar actividad metabólica, seleccionar sitio de biopsia e identificar metástasis en cuerpo entero $^{13,18-22}$.

En nuestro grupo, los pacientes más jóvenes tenían rabdomiosarcomas y tumores fibroso maligno/histiocitoma o fibrosarcoma y los niños mayores, y adultos jóvenes tumores de Ewing y osteosarcomas. Los leiomiosarcomas uterinos son raros y de post-menopáusicas, los estudiamos sólo en mujeres $>45$ años. El análisis mostró alta concordancia de captación de FDG con otros datos disponibles. La fuerza del acuerdo fue muy buena o buena en osteosarcoma, familia Ewing y alto grado. El hallazgo más relevante fue la proporción de nuevas lesiones malignas. Otro punto está relacionado con cambio en manejo y seguimiento; algunos pacientes, especialmente osteosarcomas, tuvieron FDG para seguimiento y cuando fue positivo se efectuaron imágenes anatómicas, ayudando también a dirigir biopsias. En sarcomas uterinos, la literatura es relativamente escasa, pero el FDG parece útil ${ }^{23}$.

GIST: son relativamente infrecuentes aunque corresponden a los tumores mesenquimáticos más observados en el tracto gastrointestinal (1\% los cánceres en adultos y $6 \%$ de los gastrointestinales). Aproximadamente, un tercio son malignos. El SUV ${ }_{\text {máx }}$ se asocia con índice Ki-67, tamaño del tumor, conteo mitótico y clasificación NIH; en los gástricos, parece seguro y con valor diagnóstico del potencial maligno preoperatorio. La cirugía es la terapia de elección (radio y quimioterapia convencional tienen baja utilidad). La inmunoterapia con inhibidores de la tirosin-kinasa es específica, el estudio con FDG tiene buen rendimiento pronóstico en evaluación precoz o intraterapia ${ }^{24,25}$; el PET-TC está considerado en la actualización guías NCCN recientes pero no reemplaza a la TC a pesar de concordancia de $95 \%$ entre ambas y buen valor pronóstico. Observamos utilidad tanto para estadiaje, reestadiaje y control de terapia con seguimiento metabólico.

Mesoteliomas malignos: pueden estar compuestos por elementos fibrosos epiteliales y se pueden confundir con carcinomas anaplásticos. La extensión locoregional y a distancia es frecuente, presentan alta tasa de recurrencia local. La terapia habitual es cirugía combinada o no con quimio o radioterapia. La sobrevida depende del tipo histológico siendo menor en los no epiteliales y en los con mayor grado de captación de FDG. Una experiencia reciente en 50 casos de mesotelioma pleural demostró que el FDG es una modalidad segura para diagnosticar y estimar extensión loco regional, a distancia y recurrencia ${ }^{26}$. Los pacientes presentados sólo correspondieron a tumores pleurales, que son los más frecuentes (existen peritoneales, pericárdicos y de albugínea testis). No contamos con antecedente de exposición a asbestosis. Presentaban intensa actividad pleural incluyendo el caso con pleurodesis, que se informó con componente mixto inflamatorio positivo e incerteza del compromiso tumoral. 
Meduloblastomas: son tumores embrionarios agresivos del cerebro, que se extienden localmente en forma precoz vía liquido cefalorraquídeo y raramente a distancia en espacio subaracnoideo y extraneural. Más frecuentes entre los tres y ocho años de edad y corresponden al 20\% de los tumores cerebrales infantiles (maligno más común); son poco observados en adultos. La terapia incluye cirugía agresiva con relativo buen rendimiento, aunque pueden existir secuelas neurológicas. La sobrevida con quimio y radioterapia agresiva es variable según tipo histológico y puede ser limitada si hay evidencias de progresión ${ }^{27}$. En un estudio con 22 niños y adolescentes se concluyó que la captación aumentada de FDG se correlaciona negativamente con la sobrevida; en 2/4 de ellos se observó compromiso raquídeo y a distancia esquelético ${ }^{28}$. En nuestros casos, cuando hubo sospecha de recurrencia local los estudios con FDG fueron todos positivos, con excelente concordancia con imágenes anatómicas; en la mitad, se efectuó seguimiento anual con este estudio metabólico.

Finalmente, en Chile, los tumores sólidos infantiles están cubiertos por el sistema de salud nacional y pueden realizarse cintigrafía ósea, según su tipo. El FDG podría ser una herramienta en algunos sarcomas así como está autorizado en algunos casos de linfomas ${ }^{29,30}$.

Limitaciones: es complejo efectuar seguimiento a largo plazo pues recibimos derivaciones de diversos centros. No tuvimos PET-TC disponible lo que hubiera permitido un informe más específico por localización anatómica; sin embargo, en determinados casos se efectuaron imágenes tardías para evaluar intensificación de captación en lesiones malignas y fusión con TC.

\section{Conclusiones}

El estudio PET con FDG demostró utilidad para diagnóstico y control de terapia, en sarcomas de alto grado y menos diferenciados, de adultos y niños; en la pesquisa de nuevas lesiones, lo que podría cambiar el pronóstico y manejo. En sarcomas esqueléticos, debiera considerarse en la aproximación diagnóstica y seguimiento, incluso en reemplazo del cintigrama óseo. El estudio con FDG fue también útil en tumores NSNE, especialmente respuesta a terapia específica en GIST avanzado.

\section{Referencias}

1. Rohren EM, Turkington TG, Coleman RE. Clinical Applications of PET in Oncology Radiology 2004; 231: 305-32.

2. Jofré MJ, Massardo T, González P, Canessa J, Sierralta $\mathrm{P}$, Humeres $\mathrm{P}$, et al. Uso de la tomografía de emisión de positrones PET con $\mathrm{F}^{18}$-FDG en la evaluación de lesiones pulmonares. Rev Med Chile 2005; 133: 583-92.

3. Massardo 1, ¿ofré M], Sierralta P, Canessa González P. Humeres P, et al. Análisis de 1.000 casos de estudios metabólicos en Chile con PET 18Flúor-deoxiglucosa (FDG). Rev Med Chile 2007; 135: 375-83.

4. Mochizuki T, Tsukamoto E, Kuge Y, Kanegae K, Zhao S, Hikosaka K, et al. FDG Uptake and Glucose Transporter Subtype Expressions in Experimental Tumor and Inflammation Models. J Nucl Med 2001; 42: 1551-5.

5. Martínez León MI.Meduloblastoma pediátrico, revisión y puesta al día. Radiología 2011; 53: 134-45.

6. Fletcher CDM, Unni KK, Mertens F. (Eds.): World Health Organization Classification of Tumours. Pathology and Genetics of Tumours of Soft Tissue and Bone. IARC Press: Lyon 2002.

7. O'Doherty MJ, Smith MA. PET and PET/CT Imaging in Sarcoma. In: Positron Emission Tomography. Clinical Practice. Valk PE, Delbeke D, Bailey DL, Townsend DW, Maisey MN (Eds). Springer-Verlag London Limited 2006 Ch. 17; 253-68.

8. Podoloff DA, Ball DW, Ben-Josef E, Benson AB 3rd, Cohen SJ, Coleman RE, et al. NCCN task force: clinical utility of PET in a variety of tumor types. Watl Comp Canc Netw 2009; 7: S1-26.

9. Varela S, Valenzuela P, Yacsich M, Carrasco C. Tasas de incidencia y caracterización de sarcomas en la provincia de Valdivia. Cuadernos de Cirugía 2005: 27-32 DOI:10.4206/cuad.cir.2005.v19n1-05

10. Toro JR, Travis LB, Wu HJ, Zhu K, Fletcher CD, Devesa SS. Incidence patterns of soft tissue sarcomas, regardless of primary site, in the surveillance, epidemiology and end results program, 1978-2001: An analysis of 26,758 cases. Int J Cancer 2006; 119: 2922-30.

11. Folpe AL, Lyles RH, Sprouse JT, Conrad EU 3rd, Eary JF. (F-18) fluorodeoxyglucose positron emission tomography as a predictor of pathologic grade and other prognostic variables in bone and soft tissue sarcoma. Clin Cancer Res 2000; 6: 1279-87.

12. Walter B, Czernin , Hall T, Allen-Auerbach M, Walter MA Dunkelmann S, et al. Is There a Need for Dedicated Bone Imaging in Addition to 18F-FDG PET/CT Imaging in Pediatric Sarcoma Patients? Pediatr Hematol Oncol. 2011 Nov 29. [Epub ahead of print]. 
13. Eary JF, Conrad EU. Imaging in sarcoma. J Nucl Med 2011; 52: 1903-13.

14. Hawkins DS, Conrad EU 3rd, Butrynski JE, Schuetze SM, Eary JF. [F-18]-fluorodeoxy-D-glucose-positron emission tomography response is associated with outcome for extremity osteosarcoma in children and young adults. Cancer 2009; 115: 3519-25.

15. Bastiaannet $\mathrm{B}$ Groen $\mathrm{H}$, Eager PD, Cobben DQ van der Graaf WT, Vaalburg W, et al. The value of FDG-PET in the detection, grading and response to therapy of soft tissue and bone sarcomas: a systematic review and meta-analysis. Cancer Treat Rev 2004; 30: 83-101.

16. Ioannidis JPA, Lau J. 18F-FDG PET for the Diagnosis and Grading of Soft-Tissue Sarcoma: A Meta-Analysis. J Nucl Med 2003; 44: 717-24.

17. Treglia G, Salsano M, Stefanelli A, Mattoli MV, Giordano A Bonomo D. Diagnostic accuracy of (18) FFDG-PET and PET/CT in patients with Ewing sarcoma family tumours: a systematic review and a meta-analysis. Skeletal Radiol. 2011 Nov 10. [Epub ahead of print].

18. Schulte M, Brecht-Krauss D, Werner M, Hartwig E, Sarkar MR, et al. Evaluation of neoadjuvant therapy response of osteogenic sarcoma using FDG PET. N Nuc Med 1999; 40: 1637-43.

19. Shaaban AM, Blodgett TM, Rezvani M, et al. Eds. In: Diagnostic Imaging Oncology. Musculoskeletal sites: Soft tissue sarcoma Edit. Amirsys, 2010, 2nd Ed. Section 7: 32-9.

20. Strobel K, Exner UE, Stumpe KD, Hany TF, Bode B, Mende $\mathrm{K}$, et al. The additional value of CT images interpretation in the differential diagnosis of benign vs malignant primary bone lesions with 18F-FDG-PET/CT. Eur J Nucl Med Mol Imaging 2008; 35: 2000-8.

21. Franzius C, Daldrup-Link HE, Sciuk J, Rummeny EJ, Bielack S, Jürgens $\mathrm{H}$, et al. FDG-PET for detection of pulmonary metastases from malignant primary bone tumors: comparison with spiral CT. Ann Oncol 2001; 12: $479-86$.
22. Hillner BE, Siegel BA, Shields AF, Liu D, Gareen IF, Hunt E, et al. Relationship between cancer type and impact of PET and PET/CT on intended management: findings of the National Oncologic PET Registry. J Nucl Med 2008; 49: 1928-35.

23. Sharma P, Kumar R Singh H, Eph Sharma JB, Jain SK,et al. Role of FDG PET-CT in detecting recurrence in patients with uterine sarcoma: comparison with conventional imaging. Nucl Med Commun 2012; 33: 185-90.

24. Otomi Y, Otsuka H, Morita N, Terazawa K Furutani $\mathrm{K}$, Harada M, et al. Relationship between FDG uptake and the pathological risk category in gastrointestinal stromal tumors. DMed Invest 2010; 57: 270-4.

25. Park JW, Cho CH, Jeong DS, Chae HD. Role of 18Ffluoro-2-deoxyglucose Positron Emission Tomography in Gastric GIST: Predicting Malignant Potential Preoperatively J Gastric Cancer 2011; 11: 173-9.

26. Gerbaudo VH, Mamede M, Trotman-Dickenson B, Hatabu H, Sugarbaker D]. FDG PET/CT patterns of treatment failure of malignant pleural mesothelioma: relationship to histologic type, treatment algorithm, and survival. Eur J Nucl Med Mol Imaging. 2011; 38: 810-21.

27. Riffaud L, Saikali S, Leray E, Hamlat A, Haegelen C, Vauleon E, et al. Survival and prognostic factors in a series of adults with medulloblastomas. J Neurosurg 2009; 111: 478-87.

28. Gururangan S, Hwang E, Herndon JE 2nd, Fuchs H, George T, Coleman RE. [18F] fluorodeoxyglucosepositron emission tomography in patients with medulloblastoma. Neurosurgery. 2004; 55: 1280-8.

29. Guía Clínica Linfomas y Tumores Sólidos en menores de 15 años. Garantías Explicitas en Salud 2005. http:// www.redsalud.gov.cl/archivos/guiasges/linfomasenmenores15.pdf

30. Guía clínica Linfoma en personas de 15 años y más. Santiago: Minsal, 2010 http://www.redsalud.gov.cl/portal url/item/7220fdc4340544a9e04001011f0113b9.pdf 